\title{
Study of structural chromosome abnormalities to increase the understanding of human genetic diversity: a commentary on signature of backward replication slippage at the copy number variation junction
}

\author{
Keiko Wakui \\ Journal of Human Genetics (2014) 59, 591-592; doi:10.1038/jhg.2014.83; published online 9 October 2014
}

\begin{abstract}
Tn an earlier issue of Journal of Human Genetics, Ohye et al. ${ }^{1}$ reported, at the sequence level, the chromosomal structure of a $2.8-\mathrm{Mb}$ interstitial deletion of $2 \mathrm{q}$. In this manuscript, two important implications of their report have been proposed. First, an unreported unbalanced chromosomal abnormality (UBCA) was detected with G-banding and confirmed with chromosomal microarray (CMA) and metaphase fluorescence in situ hybridization (FISH) in a subject who was a phenotypically normal adult female but suffering from recurrent miscarriage, although the relationship between UBCA and her recurrent miscarriage was uncertain. Second, the authors determined the genome structure of the junction of a microscopically visible interstitial chromosomal deletion in the subject and identified the complex repair resulting from sequence repeats with microhomology events, attributing it to a backward replication slippage mechanism. Their results suggest that such complex rearrangements of the genome may occur not only in patients but also in other phenotypically normal individuals.
\end{abstract}

Regarding chromosomal imbalances, along with recent technological advances in wholegenome analysis (WGA) using chromosomal microarray (CMA) and next-generation sequencing (NGS), humans certainly have more genomic structural variations (SVs), such as single-nucleotide variations

K Wakui is at Department of Medical Genetics, Shinshu University School of Medicine, Matsumoto, Japan

E-mail: kwakui@shinshu-u.ac.jp
(SNVs), insertion/deletion variations (indels) and copy number variations (CNVs), than suspected before. Some of these genomic changes have been identified as deleterious mutations of some diseases, termed 'pathogenic'. Various so-called 'benign' genomic changes thought to represent innocuous individual variation have also been identified. We are faced with the fact that the nature of many genomic changes is unknown; such variants are tentatively called 'variants of uncertain clinical significance (VOUS or VUS)'. Many submicroscopic CNVs (detectable only by molecular cytogenetic methods or CMA: $\mathrm{MG}^{-\mathrm{CNV}^{2}}{ }^{2}$ ) have been published in reports and databases such as Database of Genomic Variants and Phenotype in Humans using Ensembl Resources (DECIPHER) (http://decipher.sanger.ac.uk) and International Standards for Cytogenomic Arrays (ISCA) (https://www.iscaconsortium.org) of patients as well as Database of Genomic Variants (DGV) (http://dgv.tcag.ca) of healthy control samples, and this number has been increasing. However, there are larger CNVs (UBCAs or cytogenetically visible CNVs $(\mathrm{CG}-\mathrm{CNVs})^{2}$ ) that are difficult to identify even with current NGS technologies.

Individuals with UBCAs greater than several $\mathrm{Mb}$ in size usually have some kind of clinical consequences. As already identified, certain cases exhibit UBCAs, except for heteromorphism or euchromatic variants, without phenotypic manifestations. ${ }^{2,3}$ Barber $^{3}$ reviewed 130 families with directly transmitted euploid autosomal UBCAs, excluding aneuploid karyotypes and heteromorphism. The results and additional data are available as the 'The Chromosome Anomaly Collection' (http://www.ngrl.org.uk/ wessex/collection/index.htm). Among the 27 families classified in Group 1, which consisted of phenotypically unaffected parents with the same unbalanced chromosome abnormality as their unaffected children, 14 had transmitted deletions with an average size of 8.2 $\mathrm{Mb}$ (range $4.2-16.0 \mathrm{Mb}$ ). Among the 30 families in Group 2, which consisted of unaffected parents with the same autosomal imbalance as their affected children, 7 had transmitted deletions with an average size of 7.5 Mb (range 3.6-10.0 Mb). ${ }^{3}$ There may be more uncited or unpublished cases with UBCAs that fall into Group 1 or 2, or that have already been published as suspected pathogenic cases without analysis of carrier parents. In a previous study, we have also encountered a family with a $14-\mathrm{Mb}$ deletion that would be assigned to Group 2. ${ }^{4}$ More regions that might be involved in various chromosome anomalies without phenotypic consequences have been identified; these data are important and should be recognized, especially for the interpretation of prenatal screening.

When the patient is subjected to CMA and certain MG-CNVs are detected outside clinically curated regions, as references to attempt an interpretation of the phenotypic effects, it is common to compare them with online databases of CMA but less common to compare them with the data of chromosomal imbalances (CG-CNVs) or balanced chromosome rearrangements. It is recommended to 
refer to such karyotypic information when necessary, although the breakpoint and estimated abnormal size of each UBCA determined by G-banding are not always accurate or do not always match those determined with fluorescence in situ hybridization (FISH) or CMA. Thus, subjects with UBCAs should be analyzed by CMA and/or metaphase FISH, the precise abnormal regions and rearrangements should be confirmed, and such information should be added to the databases to the extent possible. Chromosomal karyotyping is the predecessor of WGA, and some chromosomal abnormalities can still be identified only by metaphase analysis. It is important to also continue maintaining conventional cytogenetic technical expertise and knowledge. $^{2}$

Regarding genome structure, constitutional structural chromosome abnormalities usually have unique breakpoints, except for some abnormalities such as Robertsonian translocations characterized by a fusion of the centromeres of two acrocentric chromosomes, the $\mathrm{t}(11 ; 22)(\mathrm{q} 23 ; \mathrm{q} 11)$ mediated by palindromic AT-rich repeats, ${ }^{5}$ and some genomic disorders (for example, Williams-Beuren syndrome and Charcot-Marie-Tooth disease type 1A), in which specific microdeletions/ duplications result from nonallelic homologous recombination mediated by low-copy repeats. 5 The majority of structural chromosome abnormalities are thought to be caused by random events leading to DNA doublestrand break repair, mainly via nonhomologous end joining or single-strand annealing.
Furthermore, recent breakpoint junction sequencing has revealed an unexpected number of complex variants associated with fork stalling and template switching, microhomology-mediated break-induced replication and the more complex chromothripsis. 6,7

Breakpoint junction sequencing has been performed to identify a responsible gene in many patients with apparently balanced chromosomal rearrangements, and the complex repair has sometimes been identified and reported. ${ }^{8,9}$ However, in particular, unique UBCAs have not been well analyzed. Thus, the precise mechanism underlying the occurrence of rare structural chromosome rearrangements remains unknown. ${ }^{1,9}$ Although we now have the types of technologies necessary for analysis of genomic SVs, even microscopically visible chromosomal rearrangements, it is still challenging. The numbers of breakpoint analyses of the samples and the development of the strategy for breakpoint junction sequencing studies have been increasing, 7,9,10 and 'Next-Generation Cytogenetic Nomenclature' was proposed.

It is desirable to identify the rearranged breakpoint junctions and the formation mechanism of more unique SVs in not only phenotypically abnormal patients but also normal individuals using every available means of genomic analysis. As sequence data accumulate, the technology and methodology, including WGA software algorithms, will improve along with an increase in the understanding of human genome diversity, including the etiology of SV-associated human diseases.

\section{CONFLICT OF INTEREST}

The author declares no conflict of interest.

1 Ohye, T., Inagaki, H., Ozaki, M., Ikeda, T. \& Kurahashi, H. Signature of backward replication slippage at the copy number variation junction. J. Hum. Genet. 59, 247-250 (2014).

2 Liehr, T. Benign \& Pathological Chromosomal Imbalances 1st edn (Oxford Academic Press: New York 2014).

3 Barber, J. C. K. Directly transmitted unbalanced chromosome abnormalities and euchromatic variants. J. Med. Genet. 42, 609-629 (2005).

4 Wakui, K., Toyoda, A., Kubota, T., Hidaka, E., Ishikawa, M., Katsuyama, T. et al. Familial 14-Mb deletion at 21q11.2-q21.3 and variable phenotypic expression. J. Hum. Genet. 47, 511-516 (2002).

5 Kurahashi, H., Bolor, H., Kato, T., Kogo, H. Tsutsumi, M., Inagaki, H. et al. Recent advance in our understanding of the molecular nature of chromosomal abnormalities. J. Hum. Genet. 54 253-260 (2009).

6 Liu, P., Carvalho, C. M., Hastings, P. J. \& Lupski, J. R. Mechanisms for recurrent and complex human genomic rearrangements. Curr. Opin. Genet. Dev. 22, 211-220 (2012).

7 Weischenfeldt, J., Symmons, O. Spitz, F. \& Korbel, J. O. Phenotypic impact of genomic structural variation: insights from and for human disease. Nat Rev. Genet. 14, 125-138 (2013).

8 Nishimura-Tadaki, A., Wada, T., Bano, G., Gough, K. Warner, J., Kosho, T. et al. Breakpoint determination of $X$ autosome balanced translocations in four patients with premature ovarian failure. J. Hum. Genet. 56 156-160 (2011).

9 Ordulu, Z., Wong, K. E., Currall, B. B., Ivanov, A. R. Pereira, S., Althari, S. et al. Describing sequencing results of structural chromosome rearrangements with a suggested next-generation cytogenetic nomenclature. Am. J. Hum. Genet. 94, 695-709 (2014)

10 Le Scouarnec, S. \& Gribble, S. M. Characterising chromosome rearrangements: recent technical advances in molecular cytogenetics. Heredity 108 75-85 (2012). 